\title{
DEVELOPING A DESKTOP APPLICATION FOR INFORMATION MANAGEMENT AND DATA RETRIEVAL SYSTEM FOR A SMALL SCALE INDUSTRY
}

\author{
${ }^{1}$ RashiSheth, ${ }^{2}$ 1Ninad Patil, ${ }^{3}$ Diksha Kosankar, ${ }^{4}$ Kishore Bhoyar \\ ${ }^{1-3}$ Under Graduate, Department of Information Technology \\ ${ }^{4}$ Professor, Department of Information Technology \\ Yeshwantrao Chavan College of Engineering, Nagpur \\ Communicating Author: Email: $\left\{{ }^{1}\right.$ rashisheth@gmail.com $\}$
}

\begin{abstract}
A small-scale industry having various departments deals with various kinds of data. The management and the flow control of data becomes a tedious job if the work is manual. So for the ease of the above purpose, an application is created on .net framework using C\# language and Server Management Studio as a back-end for database management purposes. Various modules including friendly user interfaces are created for powerful data management, data analysis, and data retrieval system. The data visualization tools help in better data analysis.
\end{abstract}

Keywords: Visual Studio, Database Management, SQL server, 2-tier architecture, Data Visualization, C\#

\section{Introduction}

Every company generates various types of data which is difficult to manage. The storage, retrieval, and manipulation of the data require lots of effort and are very time-consuming. The real problem arises when we have to use the data for extracting information and generate reports accordingly. Thus, these problems require a need to develop a system that caters to all these needs without requiring any human efforts.

\subsection{Problem Definition}

In the organization of BBNL, excel sheets were the primary source of data storage and retrieval. Thus the all work related to the data requires a lot of effort and time.

\section{Methodology}

2.1 Software Development Life Cycle

According to the project requirement, we have used iterative software development methodology as we have an iterative cycle for various modules we have.

\subsubsection{Planning and Gathering of Requirements}

The requirements and raw data gathered in this phase was used to plan the basic project approach and to conduct a software feasibility study in the operational and technical areas.

\subsubsection{Defining Requirements}

After the requirement analysis is done the next step was to clearly define and document the product or software requirements in the form of SRS (Software Requirement Specification).

\subsubsection{Designing}

A design document defines all the architectural models of the product along with its communication among the

modules and data flow representation. Based on the requirements specified in SRS a design approach for the product architecture was proposed and documented in DDS Design Document Specification.

\subsubsection{Development}

The programming code for the modules are generated as per DDS during this stage. Developing was followed according to the coding guidelines proposed by the organization.

\subsubsection{Testing}

The stage involves testing the product where product defects are reported, tracked, fixed, and retested until the product reaches the quality standards defined in the SRS.

\subsubsection{Deployment}

Deployment is the final stage of the development cycle. The product is released in the market and further according to the feedback gathered the product is enhanced with maintenance followed.

\subsection{Data Source}

The database provided by the BBNL Company is used. It mainly consists of 10000 records with 36 attributes. LG Code is taken as the primary key attribute. Figure 1 shows the snapshot of some of the attributes and figure 2 lists the attributes of the database. 


\begin{tabular}{|r|l|c|c|c|c|}
\hline Si No. & GP Name & LG Code & - & - & GP Lit \\
\hline 1 & AKOT PS HQ & 168967 & - & - & Y \\
\hline 2 & BALAPUR PS HQ & 168968 & - & - & Y \\
\hline 3 & Adgaon Kh & 168969 & - & - & Y \\
\hline. & - & - & - & - & - \\
\hline. & - & - & - & - & - \\
\hline. & - & - & - & - & - \\
\hline 10000 & Asegaon Bajar & 168999 & - & - & $Y$ \\
\hline
\end{tabular}

Fig. 1 Database

\begin{tabular}{ll|}
\hline 1) ssa & 19) New Vendors \\
2) District & 20) Length in Meters \\
3) Block & 21) Cable AT \\
4) OLT Location & 22) Date of Cable AT \\
5) GP Name & 23) GPON Eqpt AT Completed \\
6) New GP Name as per NMS & 24) Date of GPON Eqpt AT \\
7) Service Ready GPS Report & 25) Depth AT Route Length \\
8) GP LT & 26) Depth AT Complete \\
9) Date of GP LIT & 27) Date of Depth AT Complete \\
10) Fiber Tested End-to-End & 28) GPON Eqt ONT Commissioned \\
11) GPON Egpmt. (ONT) installed & 29) GPON Eqt ONT Commissioned Date \\
12) LG Code & 30) Taking over for GPON Eqpt \\
13) Updated LG Code & 31) Date of Taking over of GPON Eqpt \\
14) MAC Id & 32) Com. Cert. of OF Cable Network Commissioned \\
15) New MAC Ids as per NMS & 33) Com. Cert. of OF Cable Network Commissioned Date \\
16) Latitude & 34) RID Avallable \\
17) Longitude & 35) TOC \& MOC Certificate OFC \\
18) Vendors & 36) TOC \& MOC Certificate OFC DATE \\
\hline
\end{tabular}

Fig. 2 Attributes of Database

\subsection{Software Requirement}

The Visual Studio IDE was used to develop the application using C\# language. The database was developed on the SQL server studio platform.

Other than these the PCs in which the application needs to be installed must be in LAN connection so that they share a common centralized database while working with different modules.

\subsection{Modules}

The application starts with Login Page for any existing user and the New User page will open for registration of any new user. With the domain specified during login, the respective module will be opened. The admin login will be given access to all the modules irrespective of the domain. The specified modules that were prepared for the application were:

\subsubsection{Database Management Module}

The storage, retrieval, and modification of individual entries in the database can be done through this module. Various forms for individual tables are designed to reduce manual efforts and time.

\subsubsection{Report Generation Module}

The complete database is analyzed according to the usergenerated criteria. The module is divided into 4 sub-parts i.e. complete report, general report, often report, and extra report.

Data Visualization is an add-on feature that was added to the module to represent the information of ongoing projects in the form of pie charts.

\subsubsection{Systems and Management module}

This module caters to the need for current application form the customers regarding bandwidth requirements and dark fiber requirements. According to the requirements, a demand note is generated and issued to the customer and after the payment, an advisory note will be generated for the suppliers. The real work lies in the background where on a single click the amount needs to be calculated and a demand note or advisory note must be generated instantly without human intervention.

\subsubsection{Operations and Management}

This module deals with the inconsistency in the connections, if any. The penalty is charged if the leased cable is damaged or is unable to give the desired results. The background code calculates the cost that must be deducted as a penalty from the original bill generated.

\subsection{Back End}

We have used SQL Server Management Studio as our backend software development tool.

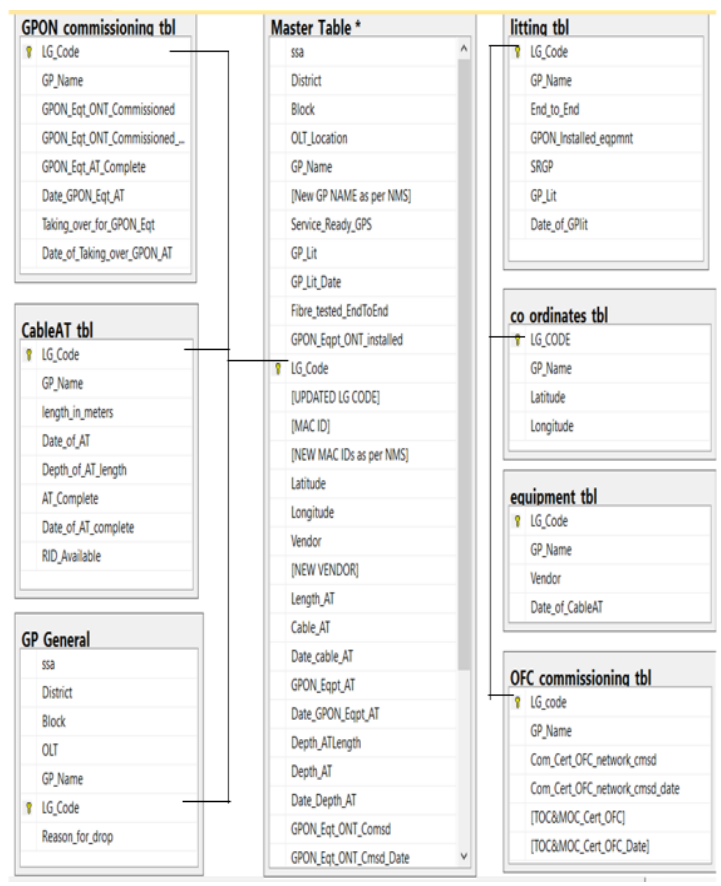

Fig.3 ER Diagram

An entire ER Diagram is designed before the actual creation of the database. Various tables are created which 
are inter-connected with each other using a primary key, 'LG_Code'. A master table comprising of all other tables, used while generation of various reports, is connected to all other child tables.

This generalization of Master Table helps in easy retrieval, storage, and manipulation of data.

\subsection{Architecture}

The architecture of the project is a basic 2-tier architecture that is similar to the client-server model. Here the client-side is deployed on the PCs of employees and the server (local) is on one centrally connected server through LAN cables to other node PCs to get access to the database.

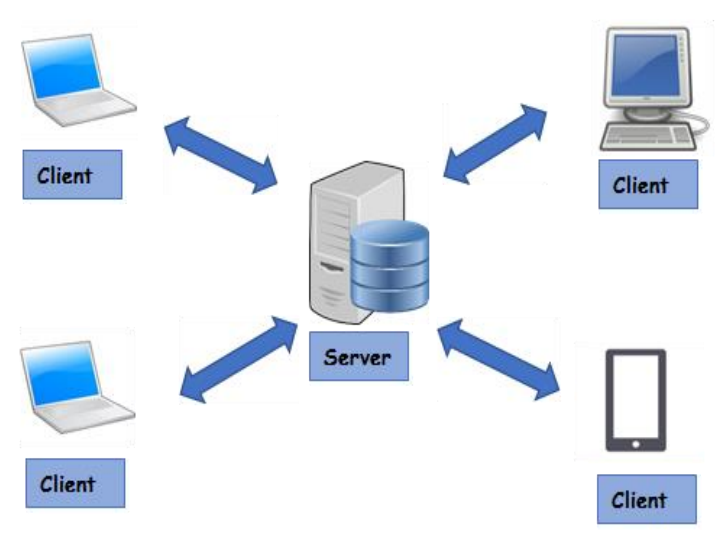

Fig.4 Architecture

\subsection{Use Case}

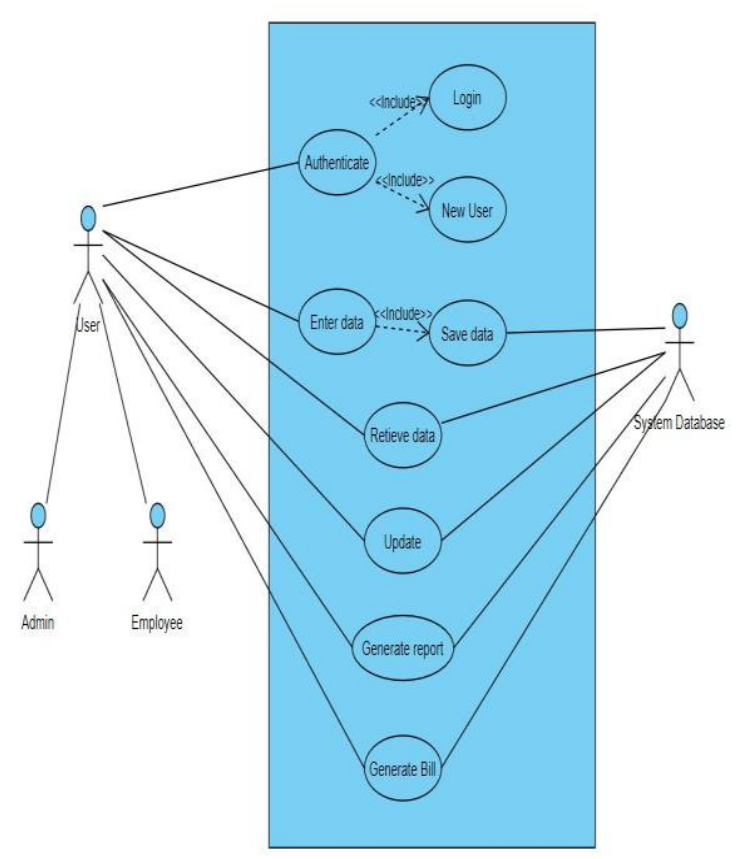

Fig.5 Use Case

\section{Literature Survey}

In this segment, we reassess the existing literature and research works done on the system automation and database management system in various fields such as college institutes, companies, inventory management, etc.

In the year 2019, V. P. Jadhav and V. M. Nandedkar performed a work, "Development of Database Management System for Small Scale Manufacturing Industry". In this paper, the author proposed a data management system to enhance information storage control. The application replaces manual paperwork and stores all information in a local MS SQL database server which can be remotely managed by company management to track the inventory information then eventually it facilitates to take a brief decision. The application can prove to be very useful in small-scale companies for saving precious time and reduces paperwork.

In the year 2017, Aishwarya Tamboli, Priti Shinde, Pravin Pariskar, Manisha Sonawane, and Chinmay Anaokar performed a work," Institute Administration Automation and Student Database Management System". In this paper, the authors here developed a method to use a personal computer to automatize the administration procedures of an educational institute and efficiently manage student's database using a Web-based application. For flexible use and lower cost, the proposed system uses a normal personal computer and a local server than a much more expensive dedicated server.

In the year 2016, Fazal Mithani, Sahista Machchhar, and Fernaz Jasdanwala performed a work," A Novel Approach for SQL Query Optimization". In this paper, the author put forth a schema that represents how some queries are going to be converted into an optimized query in a very

accurate way with a minimum amount of time and eventually it will reduce the cost of execution and increase the speed of retrieval.

In the year 2014, Haroon Shakirat Oluwatosin performed a work, "Client-Server Model". In this paper, the author explained the client-server architecture at 2-tier and 3-tier with an application and database server, and also PC as the main elements of the system. The client-server system has minimized application development time to a good extent by dividing functions into small modules by sharing information into both the client and server.

\section{Future Scope}

The proposed application is a desktop-oriented local server application. The application can be hosted on a remote server for employees that needs to work from different locations. The architecture of the application can also be converted from 2-tier to 3-tier architecture. The three-layer architecture will provide numerous benefits over client-server architecture.

\section{Conclusion}

In this paper, we have put forward a data management system application that provides an efficient data storage, retrieval, and manipulation system. A centralized data helps in better analysis and reduces time and human efforts also reduce the 
chances of possible errors. The application also provides a friendly UI which makes it easy for the employees to have their hands over the application.

\section{References}

[1] V.P. Jadhav, V.M. Nandekar," Development of Database Management System for Small Scale Manufacturing Industry", JIEM 2019.

[2] S. Amann, S. Proksch, S. Nadi and M. Mezini, "A Study of Visual Studio Usage in Practice," 2016 IEEE 23rd International Conference on Software Analysis, Evolution, and Reengineering (SANER), 2016, pp. 124134, doi: 10.1109/SANER.2016.39.

[3] F. Mithani, S. Machchhar and F. Jasdanwala, "A novel approach for SQL query optimization," 2016 IEEE International Conference on Computational Intelligence and Computing Research (ICCIC), 2016, pp. 1-4, doi: 10.1109/ICCIC.2016.7919717.
[4] Jun Tie, Jia Jin and Xiaorong Wang, "Study on application model of three-tiered architecture," 2011 Second International Conference on Mechanic Automation and Control Engineering, 2011, pp. 77157718, doi: 10.1109/MACE.2011.5988838.

[5] Dmitriy Dorofeev,ShergyShestakov," 2-tires Vs 3-Tired architecture for data processing software", ICAIT 2018.

[6] J. Armas, P. Navas, T. Mayorga, P. Rengifo and B. Arévalo, "Optimization of code lines and time of access to information through object-relational mapping (ORM) using alternative tools of connection to database management systems (DBMS)," $20172^{\text {nd }}$ International Conference on System Reliability and Safety (ICSRS), 2017, pp. 500-504, doi: 10.1109/ICSRS.2017.8272872. 